time, as was the presence of possible dimers at $6.0 \pm 0.1 \mathrm{~min}$ (small chromatographic peak). Chromatographic analysis of the same samples stored at room temperature and protected from light in a refrigerator at $4^{\circ} \mathrm{C}$ indicated the absence of a peak at $6.1 \pm 0.1$, the shift of the main peak to $8.1 \pm 0.1$, and the detection of a new chromatographic peak at $9.5 \pm 0.1$.

Conclusions The results of this study indicated the absence of aggregate formation in bevacizumab $5 \mathrm{mg} / \mathrm{ml}$ during the period of monitoring (15 days) under the two storage conditions tested. Nevertheless, they clearly indicate some kind of break down.

Acknowledgement Financial support was provided by the Project PI10/00201 (Instituto Carlos III, Ministerio de Economía y Competitividad, Spain). We also want to thank the Hospital Pharmacy Unit of the University Hospital of San Cecilio which kindly supplied all the bevacizumab samples.

No conflict of interest.

\section{TCH-026 LONG-TERM STUDY OF THE FORMATION OF AGGREGATES IN UNDILUTED CETUXIMAB $5 \mathrm{mg} / \mathrm{ml}$}

doi:10.1136/ejhpharm-2013-000276.217

'A Martínez-Ortega, ${ }^{2}$ A Salmerón-García, 'N Navas-Iglesias, 'J Hernández-Jiménez, 3J Cabeza-Barrera, 'LF Capitán-Vallvey. 'University of Granada, Analytical Chemistry, Granada, Spain; 'Baza Hospital, Hospital Pharmacy Unit, Baza, Spain; ${ }^{3}$ University Hospital San Cecilio, Hospital Pharmacy Unit, Granada, Spain

Background Cetuximab (Erbitux) is a chimeric monoclonal IgG1 antibody, an epidermal growth factor receptor (EGFR) inhibitor, given by intravenous infusion for the treatment of metastatic colorectal cancer and head and neck cancer. Cetuximab is produced in a mammalian cell line (Sp2/0) by recombinant DNA technology. Purpose To evaluate the stability of this therapeutic monoclonal antibody, i.e. cetuximab $5 \mathrm{mg} / \mathrm{ml}$ in solution for infusion, in terms of the formation of aggregates once the vial was open. The study was carried out for up to 15 days since the manufacturer only indicates chemical and physical in-use stability for up to 48 hours at $25^{\circ} \mathrm{C}$, if the solution was prepared in validated aseptic conditions.

Materials and Methods The study of the formation of the aggregates was carried out by using a size exclusion high performance liquid chromatography method with a diode array detection method (SE-HPLC-DAD. Two different storage conditions, i.e. refrigerated at $4^{\circ} \mathrm{C}$ and frozen at $-20^{\circ} \mathrm{C}$, were considered up to 15 days. Samples were characterised by chromatographic analysis immediately after the vial was opened. These chromatographic data were compared with those obtained on subsequent days. A stress study was also conducted

Results Analysis of freshly-prepared samples enabled us to characterise cetuximab chromatographically by SE-HPLC-DAD. In the corresponding chromatograms monomers were clearly detected (peak at $6.77 \pm 0.05$ minutes of retention time) while dimers or aggregates (peaks at retention times near to 6 minutes or smaller) were absent. Chromatographic analysis of the same samples stored at room temperature and protected from light in a refrigerator at $4^{\circ} \mathrm{C}$ and frozen at $-20^{\circ} \mathrm{C}$ over a 15 -day period indicate the absence of any kind of aggregates.

Conclusions The results of this study indicated the absence of the aggregate formation in cetuximab $5 \mathrm{mg} / \mathrm{ml}$ during the period of monitoring (15 days) under the two storage conditions tested.

Acknowledgment This work was supported by funds received by the Project PI10/00201 (Instituto de Salud Carlos III, Ministerio de Ciencia e Innovacion, Spain). We also thank the Pharmacy Unit of the University Hospital San Cecilio which kindly supplied all the cetuximab samples studied.

No conflict of interest.

\section{TCH-027 MEDIA FILL TO VALIDATE THE ASEPTIC PREPARATION OF SODIUM BICARBONATE INTRAVENOUS INFUSION}

doi:10.1136/ejhpharm-2013-000276.218

'E Naidovska, ${ }^{2} \mathrm{~J}$ Bajraktar, ${ }^{3} \mathrm{~B}$ Lazarova. ${ }^{\prime}$ Clinical Hospital, Department for Compounding Sterile products, Bitola, FYROM; 'Institute for Hemodialysis, Hospital pharmacy, Struga, FYROM; ${ }^{3}$ Clinical Hospital, Hospital pharmacy, Stip, FYROM

Background Sodium Bicarbonate is an alkaline agent and is indicated for treating acute or chronic metabolic acidosis. The substance is unstable and when heated in solution it gradually changes into sodium carbonate. That's why we prepared Sodium Bicarbonate Intravenous Infusion aseptically, according to the Standard Operating Procedure.

Purpose To validate the performance of aseptic processes used to produce our sterile product and to meet Good Manufacture Practice Requirements, i.e. to comply with the 'low', twice per year we are performing media fill (process simulation studies).

Materials and Methods Media fills are simulating the whole process in order to evaluate the sterility confidence of the process. Process simulations includes formulation (compounding), filtration and filling. Important factors in the process are: personnel (number, shift changes, fatigue), sterility test for the sterilised components (bottles, stoppers), filled volume per container (sufficient to wet all surfaces of the containers), frequency, media fill sizes, acceptance criteria, environmental monitoring. We select the growth medium and prepared the bulk media as the same process as routine production including filtering process and number of units (the batches is smaller than 1000). Than all units were incubated at $20-25^{\circ} \mathrm{C}$ for 14 days.

Results After the incubation period of the media filled containers they were visually examined for microbial growth. The contamination rate is zero, so, the accepted contamination rate is less then $0,1 \%$. (Contamination rate $=$ Upper confidence limit/Number of filled units $\times 100$ )

Conclusions With media fill we evaluate the aseptic assembly and operation of the sterile equipment, qualified the operators, and assess our technique, and demonstrate that the environmental controls are adequate to meet the basic requirements necessary to produce Sodium Bicarbonate Intravenous Infusion by aseptic procesing.

No conflict of interest.

\section{TCH-028 NEW BULSULFAN PROCEDURE TO IMPROVE BOTH PREPARATION AND ADMIINISTRATION}

doi:10.1136/ejhpharm-2013-000276.219

'B Dekyndt, ${ }^{2} \mathrm{~N}$ Simon, 'M Vasseur, ' ${ }^{1}$ Guerriero, ${ }^{1} \mathrm{O}$ Anzie, ${ }^{3 B}$ Décaudin, ${ }^{4} \mathrm{P}$ Odou. ${ }^{1} \mathrm{C} h r u$ De Lille, Hospital Pharmacy, Lille, France; ${ }^{2}$ Centre Oscar Lambret, Hospital Pharmacy, Lille, France; ${ }^{3}$ University Lille Nord de France, Pharmacy, Lille, France; ${ }^{4}$ UDSL EA GRIIIOT, UFR Pharmacie, Lille, France

Background When pharmacy staff is not available, nurses used to prepare diluted busulfan solution from commercial vials just before administration because of its low stability. Doing this without protection may cause occupational exposure to this cytotoxic drug.

Purpose To devise a new protocol and perform a preliminary evaluation.

Materials and Methods Literature and technical studies were performed to choose the best devices. Nurses and physicians performed a clinical evaluation using a 5-item satisfaction form.

Results Medical devices containing polycarbonate must be avoided because of the interaction with $\mathrm{N}, \mathrm{N}$-dimethylacetamide used as an excipient. The new protocol consists of an individual kit with the commercial solution packed in a syringe, an infusion bag with the 